\title{
Low-calorie sweeteners and the risk of preterm delivery: results from two studies and a meta-analysis
}

\section{Carlo La Vecchia}

Head, Department of Epidemiology, Istituto di Ricerche Farmacologiche Mario Negri, Milan and Professor, Department of Clinical Sciences and Public Health, Università degli Studi di Milano, Milan, Italy

\section{Correspondence to}

Professor Carlo La Vecchia, Department of Epidemiology, Istituto di Ricerche Farmacologiche Mario Negri, Via Giuseppe La Masa,Milan 19-20156, Italy;

carlo.lavecchia@marionegri.it

Received 1 November 2012 Revised 6 November 2012 Accepted 7 November 2012

\section{BACKGROUND}

To cite: La Vecchia C. Journal of Family Planning and Reproductive Health Care 2013, 39, 12-13.
The possible relation between low-calorie sweeteners (as well as sugar-sweetened beverages) and unfavourable pregnancy outcomes, in particular preterm delivery and low birth weight, has recently become of considerable interest, given the widespread use of low-calorie sweeteners, and the high frequency of preterm deliveries in high-income countries.

\section{NORWEGIAN COHORT DATA}

In a study from Norway, Englund-Ogge et al. $^{1}$ considered the possible relation between low-calorie-sweetened and sugar-sweetened beverages and preterm delivery using data from 60761 women in the Norwegian Mother and Child Study cohort. Overall, 3281 preterm deliveries ( $<37$ weeks) were observed, corresponding to $5.4 \%$ of all deliveries. Of these, $3.9 \%$ were late preterm deliveries (34 to $<37$ weeks), $0.8 \%$ moderately preterm deliveries (32 to $<34$ weeks) and $0.7 \%$ early preterm ( $<32$ weeks) deliveries.

There was a moderate association between frequency of use of sugar-sweetened beverages during pregnancy and the risk of preterm deliveries. As compared to women who did not drink sugar-sweetened beverages, the relative risk (RR) was 1.15 for $<1$ serving/week, 1.15 for $1-6$ servings/week, 1.25 for 1 serving/day, 1.19 for $2-3$ servings/day and 1.41 for $\geq 4$ servings/day. Allowance was made for age, education, obstetric history and tobacco but not for alcohol. Women with diabetes were excluded. Although the test for trend was significant $(p=0.008)$, a statistically significant association was already observed for sporadic use ( $<1$ serving/ week), and there was no linear dose-risk relation, since the RR was similar for $<1$ serving/week (1.15) and 2-3 servings/day (1.19).
This shed doubts on the possibility of a real association between sugar-sweetened beverages and the risk of preterm delivery.

The association with preterm deliveries was, if anything, even less consistent for low-calorie or 'artificially sweetened' beverages. Compared to non-users of artificially sweetened beverages, the RR was 1.01 for $<1$ serving/week, 1.09 for 1-6 servings/week, 1.20 for 1 serving/day, 1.01 for $2-3$ servings/day and 1.12 for $\geq 4$ servings/day. The test for trend was formally non-significant $(p=0.053)$, and there was no linear dose-risk relation; that is, the RR was 1.01 for both women reporting sporadic use $(<1$ serving/week) and for those who exhibited regular use of 2-3 drinks/day.

\section{DANISH COHORT DATA}

The issue of potential side effects of lowcalorie sweeteners in pregnancy, with specific focus on the risk of preterm delivery ( $<37$ weeks), was originally raised in 2010 by Halldorsson et al. ${ }^{2}$ from a study based on the Danish National Birth Cohort (1996-2002), including 59334 women and a total of 2739 cases of preterm births. The original report presented the results stratified by carbonated and non-carbonated drinks only, and gave a RR of 1.78 [95\% confidence interval (CI) 1.19-2.66] for drinkers of $\geq 4$ servings/day of 'artificially sweetened carbonated soft drinks". This RR estimate was, however, based on 27 cases only (i.e. less than $1 \%$ of the cases), a likely selected sub-population. This subgroup may well have other baseline characteristics that influence its risk of preterm birth, and some residual confounding is likely to be present also after multivariate analysis. There was moreover an appreciable difference between carbonated and noncarbonated drinks. The RR, in fact, was 
appreciably lower (1.29) among heavy drinkers of 'artificially sweetened non-carbonated soft drinks' (105 cases), and not materially different from that of heavy drinkers of 'sugar-sweetened non-carbonated soft drinks' (RR 1.16, 102 cases). When carbonated and non-carbonated low-calorie drinks were combined, there was no material excess risk up to 2 drinks/day (RR 1.09). ${ }^{3}$

Any possible effect of 'sweetened' versus 'artificially sweetened' drinks - as well as of carbonated versus non-carbonated drinks - on low birth weight in that study was therefore limited and inconsistent, confounded by the two types of drinks (carbonated and non-carbonated), ${ }^{4}$ as well as by residual confounding due to other likely differences in baseline characteristics of pregnant women drinking low-calorie versus sweetened, and carbonated versus non-carbonated drinks.

There is, moreover, a lack of biological consistency for any possible role of low-calorie sweeteners on the risk of low birth weight. Halldorsson et al. ${ }^{2}$ refer to a possible role of caffeine in hypertension and consequently in preterm delivery, ${ }^{5}$ but - if real - any effect of caffeine should be shared by sweetened and lowcalorie drinks. Several other standard criteria for inference in observational studies (the Hill criteria), including strength of the association, dose-risk relation, time-risk relation and consistency with other reports, also remain open to discussion. ${ }^{6}{ }^{7} \mathrm{~A}$ critical discussion of the application of the Hill criteria ${ }^{67}$ can be put forward with reference to the Danish cohort, as for most observational studies.

\section{META-ANALYSIS}

We pooled the main findings of the two studies described above using standard meta-analytic techniques. ${ }^{8}$ The Danish data were introduced in two separate strata of carbonated and non-carbonated beverages, since any possible association was apparently stronger for carbonated beverages, both sugar-sweetened and low-calorie ones.

The results of the meta-analysis are given in Table 1 . The pooled RRs in all levels of consumption were close to unity both for sugar-sweetened and for lowcalorie beverages and, most important, there was no difference in the risk estimates of sugar and lowcalorie beverages, since all the CIs largely overlap.

There is therefore no evidence that low-calorie beverages, which are US Food and Drug Administration approved for consumption during pregnancy, have an
Table 1 Combined analysis (meta-analysis) of the main findings of the studies by Halldorsson et al..$^{2}$ and Englund-Ogge et al. ${ }^{1}$ on the association between sugar-sweetened, low-calorie-sweetened beverages and the risk of preterm delivery

\begin{tabular}{lll}
\hline \multirow{2}{*}{$\begin{array}{l}\text { Beverage } \\
\text { consumption }\end{array}$} & \multicolumn{2}{l}{ Relative risk (95\% Cl) } \\
\cline { 2 - 3 } & Sugar-sweetened & Low-calorie-sweetened \\
\hline Non-users & $1^{*}$ & $1^{*}$ \\
$<1$ serving/week & $0.97(0.91-1.05)$ & $1.05(0.98-1.12)$ \\
$1-6$ servings/week & $0.93(0.87-0.99)$ & $1.09(1.02-1.16)$ \\
1 serving/day & $1.03(0.93-1.14)$ & $1.18(1.06-1.32)$ \\
2-3 servings/day & $1.06(0.96-1.16)$ & $1.14(1.02-1.26)$ \\
$\geq 4$ servings/day & $1.23(1.06-1.42)$ & $1.25(1.09-1.43)$ \\
\hline
\end{tabular}

The Halldorsson et al. study examined carbonated and non-carbonated drinks pooled from separate strata.

*Reference category.

$\mathrm{Cl}$, confidence interval.

impact on preterm delivery at any variance from that of sugar-sweetened beverages.

Funding None.

Competing interests The author has received in the past unconditional grant support from the International Sweeteners Association, Brussels, Belgium for addressing the issue of sweeteners and cancer risk.

Provenance and peer review Not commissioned; externally peer reviewed.

\section{REFERENCES}

1 Englund-Ogge L, Brantsaeter AL, Haugen M, et al. Association between intake of artificially sweetened and sugar-sweetened beverages and preterm delivery: a large prospective cohort study. Am J Clin Nutr 2012;96:552-529.

2 Halldorsson TI, Strom M, Petersen SB, et al. Intake of artificially sweetened soft drinks and risk of preterm delivery: a prospective cohort study in 59,334 Danish pregnant women. Am J Clin Nutr 2010;92:626-633.

3 Halldorsson TI. Intake of artificially sweetened soft drinks and risk of preterm delivery. Am J Clin Nutr 2010;92:1540; author reply 1540-1542.

4 La Vecchia C. Intake of artificially sweetened soft drinks and risk of preterm delivery. Am J Clin Nutr 2010;92:1540.

5 Winkelmayer WC, Stampfer MJ, Willett WC, et al. Habitual caffeine intake and the risk of hypertension in women. JAMA 2005;294:2330-2335.

6 Bursey RG, Watson ML. Intake of artificially sweetened soft drinks and risk of preterm delivery. Am J Clin Nutr 2010;92:1277-1278: author reply 1278-1280.

7 Hill AB. The environment and disease: association or causation? Proc R Soc Med 1965;58:295-300.

8 Greenland S. Quantitative methods in the review of epidemiologic literature. Epidemiol Rev 1987;9:1-30. 